\title{
OPTICAL PROPERTIES AND MICROSTRUCTURAL CHANGES OF HARD DENTAL TISSUES IN GASTRO-ESOPHAGEAL REFLUX DISEASE PATIENTS
}

\author{
OLGA HILDA ORASAN ${ }^{a}$, ANDREA MARIA CHISNOIU ${ }^{b}$ *, \\ MONICA LAURA DASCĂLU (RUSU) ${ }^{c}$, OVIDIU PĂSTRAV ${ }^{d}$, \\ MIHAELA PĂSTRAV ${ }^{e}$, MARIOARA MOLDOVAN ${ }^{f}$, RADU CHISNOIU ${ }^{d}$
}

\begin{abstract}
Dental erosion is a frequent complication of patients with gastro-esophageal reflux disease. Loss of superficial enamel can be observed by a color change towards yellow and increased translucency of the teeth. The aim of our study was to analyze salivary parameters and dental erosion status in patients with gastro-esophageal reflux disease, and to evaluate optical properties (color parameters variation) and microstructural modifications (using atomic force microscopy) of eroded compared to healthy dental structures. The association of dental erosion and gastro-esophageal reflux disease was significant. Variations of $\Delta \mathrm{E}$ are higher than general average variations of $\Delta \mathrm{L}$ in these patients.
\end{abstract}

Keywords: gastro-esophageal reflux disease, dental erosion, color parameters variation, atomic force microscopy

a $4^{\text {th }}$ Medical Department, Faculty of Medicine, University of Medicine and Pharmacy "Iuliu Hatieganu", 18 Republicii Street, 400015, Cluj-Napoca, Romania.

b Department of Prosthetic Dentistry, Faculty of Dental Medicine, University of Medicine and Pharmacy "Iuliu Hatieganu", 32 Clinicilor Street, 400006, Cluj-Napoca, Romania.

* Corresponding author: ANDREA CHISNOIU, e-mail: dr.chisnoiu@yahoo.com

${ }^{\mathrm{C}}$ Department of Preventive Dentistry, Faculty of Dental Medicine, University of Medicine and Pharmacy "Iuliu Hațieganu", 32 Clinicilor Street, 400006, Cluj-Napoca, Romania.

'Department of Odontology and Oral Pathology, Faculty of Dental Medicine, University of Medicine and Pharmacy "Iuliu Hațieganu", 33 Motilor Street, 400001, Cluj-Napoca, Romania.

e Department of Orthodontics and Orofacial Orthopaedics, Faculty of Dental Medicine, University of Medicine and Pharmacy "Iuliu Hațieganu", 33 Motilor Street, 400001, Cluj-Napoca, Romania.

f "Babes Bolyai" University -"Raluca Ripan" Chemistry Research Institute, 30 Fântânele Street, 400294, Cluj-Napoca, Romania. 


\section{INTRODUCTION}

Gastro-esophageal reflux disease (GERD) is the most frequent esophageal pathology, a chronic disease with high morbidity risk and potential mortality by its complications [1].

A positive correlation has been identified between excessive acid exposure and chemical dissolution of dental structures, assessed by esophageal $\mathrm{pH}$ monitoring [2-4]. The enamel dissolution is a fast process that produces the extensive diffusion of protons layer in solution $[5,6]$.

Hydrochloric acid from the gastric contents is responsible for the demineralization of dental hard tissues and release of matrix metalloproteinase from the dentin. The clinical effect, produced by the opening of dentinal tubules, is the apparition of cervical dentinal sensitivity, as a result of dynamic changes of dentinal fluids and dental tissues loss $[7,8]$.

Tooth wear is usually assessed using visual scoring systems. These methods lack criteria for distinguishing stages of erosion limited to the enamel. Thus, erosion is mostly diagnosed at a severe stage and is difficult to monitor $[9,10,11]$. Currently two quantitative assessment methods have been described: profilometry and ultrasound. Loss of superficial enamel can be observed by a color change of the teeth towards yellow. Reducing enamel thickness will increase translucency, making the underlying dentin more visible [12-14]. We hypothesized that a useful tool for detecting and monitoring dental erosion might be represented by this color change.

Most data from research about color in dentistry are obtained under the CIEL ${ }^{*}{ }^{*} b^{*}$ (CIE: Commission Internationale de l'Eclairage; International Commission on Illumination) system, and these are reported by corresponding symbols L* (luminosity), $a^{*}$ (green-red color coordinate), $b^{*}$ (blue-yellow color coordinate), $\mathrm{C}^{*}$ (shade), $\mathrm{h}$ (saturation) and $\Delta \mathrm{Eab}$ (global color difference). The formula for $\Delta \mathrm{Eab}$ is:

$$
\Delta \mathrm{Eab}=\left[\left(\Delta \mathrm{L}^{*}\right)^{2}+\left(\Delta \mathrm{a}^{*}\right)^{2}+\left(\Delta \mathrm{b}^{*}\right)^{2}\right]^{1 / 2},
$$

where $\Delta \mathrm{L}^{*}, \Delta \mathrm{a}^{*}, \Delta \mathrm{b}^{*}$ represent the difference between two values of the same parameter. The values of color difference $\Delta \mathrm{E}_{\mathrm{ab}}$ can offer a clear image of the "matching" degree between two samples or teeth $[15,16]$.

SEM microscopy performs qualitative analysis of the enamel surfaces changes under erosive processes. SEM microscopy determines the extension of the affected enamel surfaces regarding the alteration degree of enamel prisms accordingly to SEM images [17].

This study aimed to analyze salivary parameters $(\mathrm{pH}$ and buffering capacity) and dental erosion status in patients with GERD symptoms. The research was also focused on the evaluation of optical properties (color parameters variation) and microstructural changes (using atomic force microscopy- AFM) of eroded compared to healthy dental structures. 


\section{RESULTS AND DISCUSSION}

The degree of erosion in patients with GERD (group 1) was higher than in controls. Most of the patients in the non-GERD group $(89.5 \%, n=43)$ presented a BEWE score lower than 9. In the GERD group, 63.6\% $(n=14)$ of patients presented a BEWE score higher than 9 (Fig. 1).

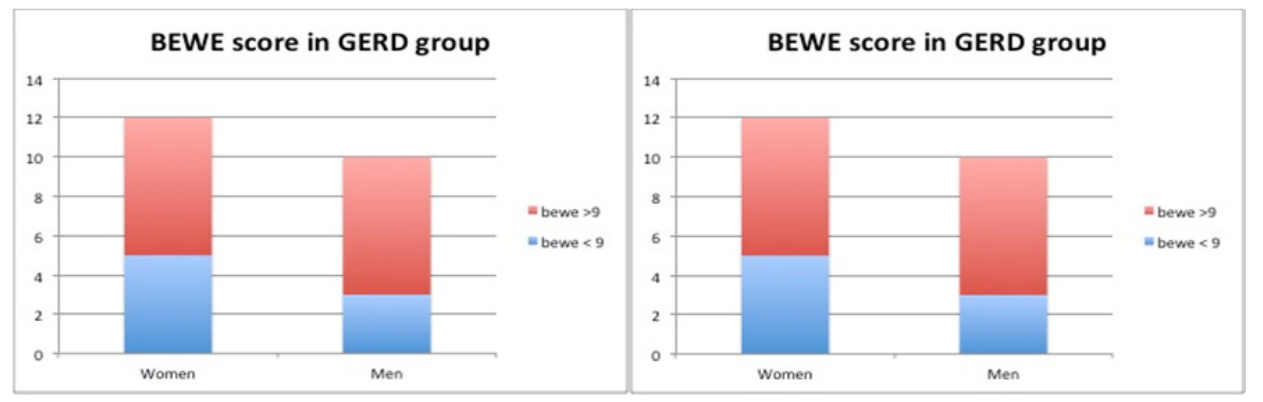

Figure 1. BEWE score in the GERD group vs the non-GERD group (BEWE: Basic Erosive Wear Examination; GERD: gastro-esophageal reflux disease)

The risk for developing dental erosion was mostly absent or low among non-GERD patients. Patients in the GERD group more frequently presented a medium or high risk for dental erosion $(p=0.032)$ (Fig. 2).

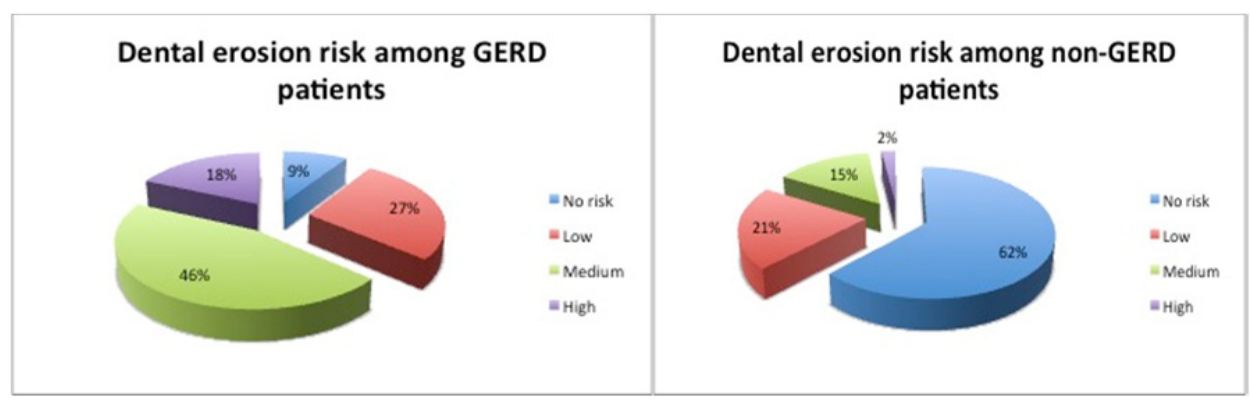

Figure 2. Dental erosion risk in the GERD group vs the non-GERD group (GERD: gastro-esophageal reflux disease)

There were no $\mathrm{pH}$ differences between the 2 groups $(p=0.36)$. Salivary buffering capacity in patients with GERD showed lower values than in controls $(p=0.048)$. The mean buffering capacity in the GERD group was 8. A significant direct correlation was identified between the BEWE score and buffering capacity $(p=0.003)$. For each type of substrate taken into study, the 2 groups of teeth, respectively, the average values of $\Delta \mathrm{E}$ and 
O. H. ORASAN, A. M. CHISNOIU, M. L. DASCĂLU (RUSU), O. PĂSTRAV, M. PĂSTRAV, M. MOLDOVAN,

R. CHISNOIU

$\Delta \mathrm{L}$ were calculated. The results and the statistical analysis for measuring color parameters using the Vita Easyshade spectrophotometer are presented in Tables 1 and 2. The calculation of the two parameters tracked by us was made according to the above formulas, and statistical calculation was performed based on samples taken into work.

Table 1. Calculated $\Delta \mathrm{E}$ and $\Delta \mathrm{L}$ values in the extracted teeth samples from healthy (non-GERD) patients and GERD patients

\begin{tabular}{lcc}
\hline Samples & $\Delta \mathbf{E}$ & $\Delta \mathbf{L}$ \\
\hline \multirow{3}{*}{ Non-GERD } & 1.70 & 1.30 \\
\cline { 2 - 3 } & 0.70 & -0.70 \\
\cline { 2 - 3 } & 1.89 & -1.60 \\
\hline \multirow{3}{*}{ GERD } & 3.01 & 2.90 \\
\cline { 2 - 3 } & 2.92 & 2.70 \\
\cline { 2 - 3 } & 10.87 & 0.70 \\
\cline { 2 - 3 } & 14.81 & -5.80 \\
\cline { 2 - 3 } & 10.04 & 2.90 \\
\hline
\end{tabular}

$\Delta \mathrm{E}$ : color differences; $\Delta \mathrm{L}$ : luminosity differences; GERD: gastro-esophageal reflux disease

Table 2. t-Test for the GERD group

\begin{tabular}{lcc}
\hline \multicolumn{3}{c}{ t-Test: Paired Two Sample for Means } \\
\hline GERD & $\Delta \mathrm{E}$ & $\Delta \mathbf{L}$ \\
\hline Mean & 2.04270652 & 0.92 \\
\hline Variance & 0.90918759 & 4.052 \\
\hline Observations & 5 & 5 \\
\hline Pearson Correlation & 0.486657501 & 0.083285954 \\
\hline Hypothesized Mean Difference & 0 & 0 \\
\hline Df & 4 & 4 \\
\hline $\mathbf{t ~ S t a t}$ & -3.858925877 & -2.248726175 \\
\hline $\mathbf{P}$ (T<=t) one-tail & $\mathbf{0 . 0 0 9 0 8 1 9 3 1}$ & $\mathbf{0 . 0 4 3 8 8 4 4 6 3}$ \\
\hline $\mathbf{t}$ Critical one-tail & 2.131846786 & 2.131846786 \\
\hline $\mathbf{P}$ (T<=t) two-tail & 0.018163862 & 0.087768926 \\
\hline $\mathbf{t}$ Critical two-tail & 2.776445105 & 2.776445105
\end{tabular}

$\Delta \mathrm{E}$ : color differences; $\Delta \mathrm{L}$ : luminosity differences; GERD: gastro-esophageal reflux disease 
Statistics applied to color parameters $(\Delta E)$ in the healthy (nonGERD) and GERD groups showed significant changes for eroded dental structures on the analyzed surfaces. The results registered for the morphology and topography of dental enamel on teeth extracted from healthy patients show the surface of enamel with uniform projections of smaller sizes, without a specific alignment (Figure 3) .
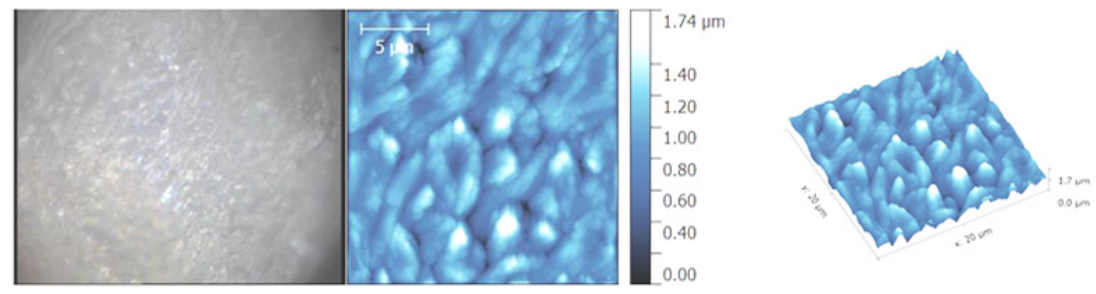

Figure 3. Enamel aspect from a healthy patient: a-optical image, b- two-dimensional AFM image; c- three-dimensional AFM image. Scan parameters: scanned area aria $20 \times 20 \mu \mathrm{m}^{2}, 256$ × 256 pts, scanning frequency $1 \mathrm{~Hz}$, FB 1, scanning speed $97.85 \mu \mathrm{m} / \mathrm{s}$, set point

AFM images registered on teeth from patients with GERD, have revealed irregular surfaces of higher sizes, with deep porosities between dimples (Figure 4).
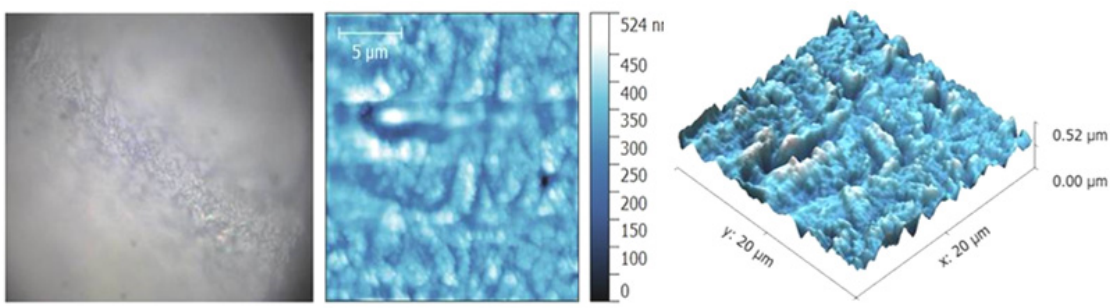

Figure 4. Enamel aspect from GERD patients: a-optical image, b- two-dimensional AFM image; c- three-dimensional AFM image. Scan parameters: scanned area aria $20 \times 20 \mu \mathrm{m}^{2}, 326 \times 326 \mathrm{pts}$, scanning frequency $1 \mathrm{~Hz}$, FB 1, scanning speed $97.85 \mu \mathrm{m} / \mathrm{s}$, set point

The association of dental erosion and GERD in our study is significant. Similar results were obtained by Fatemeh Farahmand et al. [19], who found that 53 of $54(98.1 \%)$ GERD patients and 11 of $58(19.0 \%)$ controls had dental erosions ( $p<0.0001)$. Jarvinen et al. [20] examined 109 patients with upper gastrointestinal symptoms and found only $6.4 \%$ to have erosion, while Meurman et al. [21] found $26.2 \%$ out of 107 patients diagnosed with gastro-esophageal reflux to have dental erosion. For Pace 
F et al. [22], the median prevalence of dental erosion in GERD patients was $24 \%$, with a large range (5-48\%), and the median prevalence of GERD in adult patients with dental erosion was $32.5 \%$ (range $21-83 \%$ ), while in pediatric patients it was $17 \%$ (range 14-87\%).

Lower $\mathrm{pH}$ values in GERD patients according to other studies (1921 ) confirm the higher risk of dental erosion in this condition.

There are many factors that may contribute to the variation in the reported CIELab values in different studies. Of course, there may be a considerable variation in the actual tooth colors of the subjects or extracted teeth studied. In addition, the measurement technique and circumstances may introduce differences [23]. Spectrophotometers are extremely sensitive devices that can detect minimal color changes which are not really observable clinically. For these reasons, it was decided to use spectrophotometric analysis in the current study for evaluation and determination of color changes in the teeth by using the parameters CIE L * $a{ }^{*} b{ }^{*}$ and delta $E$ and also, the closest shades using the intraoral Vita Easyshade spectrophotometer [24-25]. Regarding the results of our study on the extracted teeth, we found the following: $\Delta \mathbf{E}$ variations are higher than general average $\Delta \mathrm{L}$ variations in the case of GERD patients.

AFM evaluation of hard dental structures has proven the presence of increased enamel micro-porosities in GERD patients. Hydrochloric acid from gastric juice can affect not only the surface but also the internal structure of enamel [26].

\section{CONCLUSIONS}

It was concluded that the high degree of dental erosion must be a consequence of acid attack and reduced salivary buffering capacity in patients with GERD symptoms. Tooth color variations might represent a useful tool for monitoring dental erosion in GERD patients.

\section{EXPERIMENTAL SECTION}

The research was divided into two parts: the first section included a clinical evaluation of dental structures and salivary parameters in patients with and without GERD symptoms. The second section focused on the evaluation of optical properties of eroded compared to healthy dental structures.

The first part of the study consisted of the evaluation of 70 patients (mean age $45 \pm 15$ years) attending an internal medicine service in ClujNapoca, of which 48 were healthy subjects (group 1) and 22 presented GERD symptoms (group 2). GERD symptoms were evaluated based on a 
valid questionnaire. For dental erosion, BEWE (Basic Erosion Wear Examination) was used. Oral clinical examinations were performed and DE was evaluated using the BEWE (Basic Erosive Wear Examination) Index [17]. The buccal $(B)$, palatal $(P) /$ /ingual $(L)$ and occlusal $(O) /$ incisal (I) surfaces of all teeth were examined, and the highest score in each sextant was noted. The final score was obtained by summing up the values of all sextants; it was used to assess the risk for DE development in each patient. Dental examination was conducted by trained investigators.

The BEWE score quantifies the DE risk as follows: < 3: no risk; 3-8: low risk; 9-13: medium risk; $\geq 14$ : high risk.

Analysis of $\mathrm{pH}$ (5-5.8 highly acid, 6-6.6 moderately acid, 6.8-7.8 healthy saliva) and buffer capacity (0-5 very low, 6-9 low, 10-12 normal) of the saliva was also performed and was recorded using specific salivary tests (GC Saliva Tests).

The second part of the study was conducted using 32 decay-free human teeth, extracted for periodontal reasons. The study was conducted on two groups as follows:

Group 1 - 16 teeth were obtained from patients with GERD. The teeth were free of decay or other dental disorders and had indications for dental extraction due to periodontal disease.

Group 2 - 16 teeth were collected from healthy patients, free from GERD. The procedures for extraction and preservation of the teeth were carried out after patients signed an informed consent.

All teeth used for study were washed right after extraction, professionally sanitized by scaling and brushing of crown and root surfaces, and kept in artificial saliva until use. For their better handling, they were embedded in self-curing acrylic resin (Duracryl Plus, Spofa Dental, Czechia; shade 0). Subsequent to incorporation, the crown and the cervical third of the root (non-embedded portion) were indented with a high consistency silicone material, in order to obtain the patterns necessary to achieve some polyethylene caps. The latter were made by thermoforming, with the closest adaptation to the coronal surface. Making the caps had a double role - to allow the registration of color parameters, with the Vita Easyshade spectrophotometer, as well as to allow a more precise adjustment of the spectrophotometer loop to the dental surface. As such, for each tooth, on the vestibular and oral surface of the cap, holes were made with a diameter equivalent to the diameter of the loop of the Vita Easyshade spectrophotometer (Vita-Zahnfabrik).

For measuring the color parameters we used a Vita Easyshade (Vita-Zahnfabrik) spectrophotometer consisting of three essential elements: a source of light, a means of directing the light reflected by the object, and 
O. H. ORASAN, A. M. CHISNOIU, M. L. DASCĂLU (RUSU), O. PĂSTRAV, M. PĂSTRAV, M. MOLDOVAN, R. CHISNOIU

a spectrophotometer designed to determine the intensity of the received light depending on wave length. A major advantage of this device is to determine dental color regardless of lighting conditions, thanks to its own source of light.

The measurements were performed globally, initially immediately after extraction and after 5 days of immersion in artificial saliva, and aimed at determining the parameters of color in absence of the oral environment. The measurements were made on the vestibular and oral surfaces of each tooth, and for each area four measurements were made. The arithmetic average of measurements was used for calculating the difference in color using the formula [1]. Average values of luminosity differences $\Delta L^{*}$, chromatic parameters $\Delta \mathrm{a}^{*}$ and $\Delta \mathrm{b}^{*}$, color differences $\Delta \mathrm{E}^{*}$ for each tested substance were statistically analyzed using the ANOVA test and test for the comparison of two groups.

The retrieval protocol of color parameters with the spectrophotometer was the following: for the extracted teeth: the reading end of the spectrophotometer was applied perpendicular to and centered mesio-distally on the vestibular surface, with its margin $1 \mathrm{~mm}$ from the cervical line.

The AFM images were taken over with a commercial microscope Ntegra Spectra (NT-MDT, Russia), at room temperature, in the air, in intermittent contact mode (semi contact), with rectangular cantilever of silicon with reflective surface of Au (NSG30-A, NT-MDT), normal spring constant $\mathrm{k}=40 \mathrm{~N} / \mathrm{m}$, resonant frequency $240-440 \mathrm{kHz}$, peak radius $<10$ $\mathrm{nm}$. After acquisition, the images were processed using the Nova v1.1.0.1837 (NT-MDT) program.

\section{STATISTICS}

All data were analyzed using SPSS Statistics version 21 software. Statistical comparisons were performed using Student's t-test. The values were expressed as mean \pm standard deviation (SD). A P value $<0.05$ was considered statistically significant.

\section{ACKNOWLEDGMENTS}

This work was funded by the Romanian Ministry of Education and Research, National project 142PED/2017. 


\section{REFERENCES}

1. N. Vakil, S.V. van Zanten, P. Kahrilas, J. Dent, R. Jones, American Journal of Gastroenterology, 2006, 101, 1900.

2. E. Ness-Jensen, A. Lindam, J. Lagergren, K. Hveem, Gut, 2012, 61, 1390.

3. S. Bruley Des Varannes, L. Marek, B. Humeau, M. Lecasble, R. Colin, Gastroenterology Clinical Biology, 2006, 30, 364.

4. R.C. Orlando. American Journal of Medical Science, 2003, 326, 274.

5. D.W. Bartlett, D.F. Evans, B.G. Smith, Journal of Oral Rehabilitation, 1996, 23, 289.

6. D. Bartlett, C. Ganss, A. Lussi, Clinical Oral Investigations, 2008, 12, 65.

7. B.G. Smith, J.K. Knight, British Dental Journal, 1984, 156, 435.

8. G.K. Johnson, J.E. Sivers, Clinical Preventive Dentistry, 1987, 9, 12.

9. N. Schlueter, C. Ganss, S. De Sanctis, J. Klimek, European Journal of Oral Science, 2005, 113, 505.

10. M.C. Huysmans, J.M. Thijssen, Journal of Dentistry, 2000, 28, 187.

11. M. Moldovan, L.S. Dumitrescu, C. Prejmerean, D. Dudea, V. Popescu, Materiale Plastice, 2010, 47, 421.

12. C. Louwerse, M. Kjaeldgaard, M.C. Huysmans, Journal of Dentistry, 2004, 32, 83.

13. J.J. Ten Bosch, J.C. Coops, Journal of Dental Research, 1995, 74, 374.

14. R.D. Douglas, Journal of Prosthetic Dentistry, 1997, 77, 464.

15. R.D. Paravina, G. Majkic, F. Imai, J. Powers, Journal of Prosthodontics, 2007, 16, 269.

16. A. Baltzer, V. Kaufmann-Jinoian, Quintessenz Zahntechnik, 2004, 30, 726.

17. J. Field, P. Waterhouse, M. German, Journal of Dentistry, 2010, 38, 182.

18. A. Lussi, T. Jaeggi. "L'erosion dentaire. Diagnostic, évaluation du risque, prévention, traitement." Paris, Berlin, Chicagio: Quintessence International DL, 132, 2012

19. F. Farahmand, M. Sabbaghian, S. Ghodousi, N. Seddighoraee, M. Abbasi, Gut Liver, 2013, 7, 278.

20. V. Jarvinen, J.H. Meurman, H. Hyvarinen, I. Rytomaa, H. Murtomaa, Oral Surgery Oral Medicine Oral Pathology, 1988, 65, 298.

21. J.H. Meurman, J. Toskala, P. Nuutinen, E. Klemetti, Oral Surgery Oral Medicine Oral Pathology, 1994, 78, 583.

22. F. Pace, S. Pallotta, M. Tonini, N. Vakil, G. Bianchi Porro. Aliment Pharmacology Therapeutics, 2008, 27, 1179.

23. A. Dozic, C.J. Kleverlaan, I.H. Aartman, A.J. Feilzer, Dental Materials, 2005, 21, 187.

24. R.R. Seghi, W.M. Johnston, W.J. O'Brien, Journal of Dental Research, 1989, $68,1755$.

25. D. Dudea, M. Moldovan, L. Silaghi Dumitrescu, H. Colosi, A. Botos, A. Irimie, C. Alb, Studia UBB Chemia, 2010, 1, 65.

26. A.M. Bargan, G. Ciobanu, C. Luca, E. Horoba, Studia UBB Chemia, 2013, 58, 137. 